\title{
Research on Bridge structural Health Monitoring System Based on WSN and Mobile Agent
}

\author{
Yu A-long, Dai Jin-qiao, Yang Wei-wei \\ School of Physics and Electronic Electrical Engineering, Huaiyin Normal University Huaian, \\ Jiangsu 223300, PR China \\ yal@hytc.edu.cnl, djq@hytc.edu.cn, yww@163.com
}

\begin{abstract}
Keywords: Wireless sensor network, Mobile Agent, Structural health monitoring, ZigBee, GPRS Abstract. The technologies of wireless sensor networks (WSNs) and mobile agent are applied because of the large and complicated bridge structure, large amounts of sensors and multiple damage forms. The paper takes the actual bridge structure as the research object, adopting CC2530 as the core of wireless sensor networks nodes to collect relevant bridge structure data. The mobile Agent migrates and accesses to network nodes to complete collection and processing of the nodes data by its mobility. Data are transferred to the gateway node for processing through ZigBee wireless communication protocol. Through the GPRS wireless communication mode, eventual data are sent to monitoring center to determine the health status of bridge. The results show that the system meets the design requirements. The monitoring data is reliable as well, which states that the system is worth being promoted and implemented.
\end{abstract}

\section{Introduction}

Bridges inevitably suffer from all kinds of damage during implementation. These damages will reduce the structural bearing capacity and security. Therefore, the construction and maintenance of bridge has become an important part of national infrastructure construction. In the early days, wired health monitoring technology [1-2] has been widely used to monitor damages through arranging sensors in the key parts of bridge. But it is found that the wired health monitoring system is very expensive to install and maintain in the practical application. It is difficult to complete the wiring in the special parts and vulnerable to be damage from the environment. The transmission distance is limited by the length of the wiring [3]. Besides, it may block the traffic during remolds. Hence, we should find more effective solutions to solve these problems.

Wireless sensor network is used to achieve sensor node deployment and networking. Not only solved the problem of wiring, but also enlarged transmission distance. Besides, the system is stable and less susceptible to environmental damage. Through the processing and analysis of data collected by various sensors in real time, timely feedback of the working status and health status of bridge is the best solution to realize the online monitoring of bridge health. Wireless sensor network [4] is a kind of measurement and control network system [5] located in unsupervised monitoring area, which is composed of a large number of same or different wireless sensor nodes. Due to the dense nodes, energy storage capacity, wireless communication and computing capability are all limited, it is difficult to maintain. Furthermore, there is a lack of collaboration between nodes. Thus, there is still a problem of inefficiency.

In recent years, agent ideology has been applied to wireless sensor networks. Mobile agent can acquire, analyze and process data without user supervision. It pays more attention to the collaboration between nodes and subnets and has many advantages such as reducing network loads, strong adaptability and sensitive reaction. Hence, many scholars focus on the application of mobile agents in the field of wireless sensor networks. The paper focuses on the combination of wireless sensor networks and mobile agents in the bridge health monitoring application. 


\section{WSN and Mobile Agent}

\section{A. WSN}

Wireless Sensor Network (WSN) is a new type of network structure without center nodes, which consists of a number of micro-wireless sensor nodes with same or different functions deployed in the monitoring area [5]. Wireless Sensor Network forms a multi-hop self-organized network system through wireless communication, which combines sensor technology, wireless communication technology, embedded technology, distributed information processing technology and others. As a ubiquitous sensing technology, wireless sensor network is widely implemented in military, environmental science, space exploration, commercial applications and other fields [6].

The typical wireless sensor network system is mainly composed of target object, wireless sensor nodes, wireless sensing area, Sink node, the Internet or satellite communication, user interface and so on. The architecture of wireless sensor network is shown in figure 1.

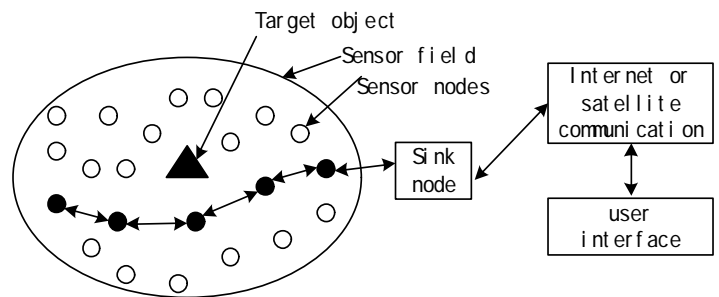

Fig. 1. Architecture of wireless sensor network

\section{B. Mobile Agent}

Mobile agent can effectively combine distributed technology and artificial intelligence technology, and perform the task assigned by users in the form of code. In addition to the basic property of intelligent agent, the most important feature is mobility. It can autonomously migrate from one node to another node in a heterogeneous network. It is also a program which can interact with other agents or resources. Actually, it is a hybrid of agent technology and distributed computer technology [7]. Mobile agent has the following four advantages [7]. First, mobile agent preserves data on the local node by technical specifications and transfers the integrated process (code) to data node. So that mobile agent is less dependent on the middle link of network transmission, and directly faces the resources to be accessed. Thereby, a large number of data transmissions in network have been avoided. Network load and dependence of system on the network bandwidth have also been reduced. Second, once the agent was generated, it can move intelligently in the network without depending on the specific source node. When a source node broken down or need to be shut down, a warning can be made to the mobile agent whom is running in the node. Then the agent can move to other nodes in the network in a short period and continue to operate normally. Third, mobile agent can support discontinuous calculation. When the network is stable, mobile agent can be sent from source to destination and return the result when the network is re-established. Besides, it can move among different nodes asynchronously and has a strong self-executing function. Fourth, mobile agent has a strong ability to dynamically adapt to the environment. The performance of agent can be adjusted according to the environmental situation.

Therefore, the introduction of mobile agent technology not only narrows the cooperative relationship between nodes and subnets, but also reduces a large number of redundant data in the network transmission.

\section{Applications of Mobile Agent in wireless sensor network}

These features and advantages of mobile agent can effectively improve the performance of wireless sensor network, which is very suitable for the application in the field of wireless sensor network. Therefore, we propose a structural model of wireless sensor network based on mobile agent, as shown in figure2. 


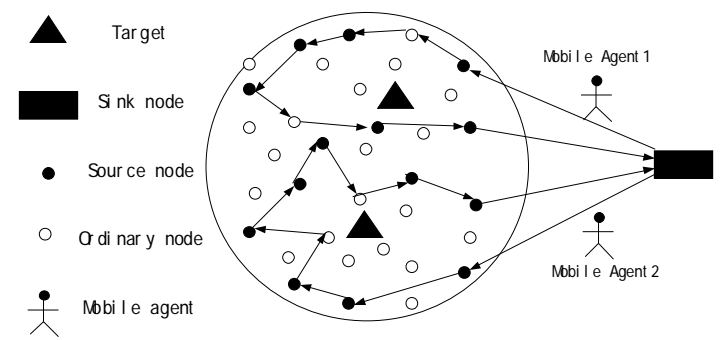

Fig. 2. Architecture of wireless sensor network based on Mobile Agent

Different from wireless sensor network architecture, this architecture adds the mobile agent entity based on the four basic objects such as target object, wireless sensor node, wireless sensor area and Sink node. Mobile agent migrates along a certain path and visits network nodes without user intervention. Then mobile agent acquires, analyzes, and processes data voluntarily. Wireless sensor network is application-oriented and data-centric. The application scenario [8] of mobile agent in the network is as follows. The Sink node, as the controller of network query and recipient of sensing data, has more resources. It is also the creator and recycler of mobile agent. The sensing data of sensor node is no longer converged to the Sink node, but completes the collection and processing of node data through migration and visit of mobile agent in the network. The flow chart of data collection based on the mobile agent [9] is shown in figure 3.

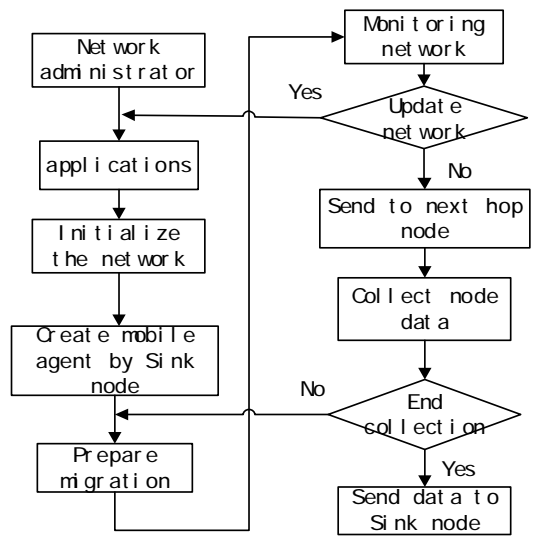

Fig. 3. Flow chart of data collection based on the Mobile Agent

\section{Bridge structural health monitoring based on WSN and Mobile Agent}

\section{A. System structure}

The system consists of wireless sensor network area and gateway area [10].The wireless sensor network area contains a number of monitoring areas and a base station node. Each monitoring area has a number of wireless sensor network nodes and a Sink node. The wireless sensor network uses the central processor to collect the perception data in the monitoring area. By utilizing the mobility of mobile agent and other characteristics, the collaborative negotiation between nodes in the monitoring area and between the monitoring areas, the collection and arrangement of bridge monitoring related data can be completed. Through the Zigbee wireless communication protocol, the data arranged by base station are sent to gateway equipment for comprehensive processing. Then the processed data are sent to remote control center by Internet, GPRS or other ways. In the remote control center, the monitoring staff can observe the status of monitoring system at any time and send monitoring command to sensor node and gateway through host computer software. When the parameter of a monitoring area exceeds the preset threshold, the gateway equipment will immediately send an alarm signal to the control center. The composition of bridge structural health monitoring system based on WSN and Mobile Agent is shown in figure 4. 


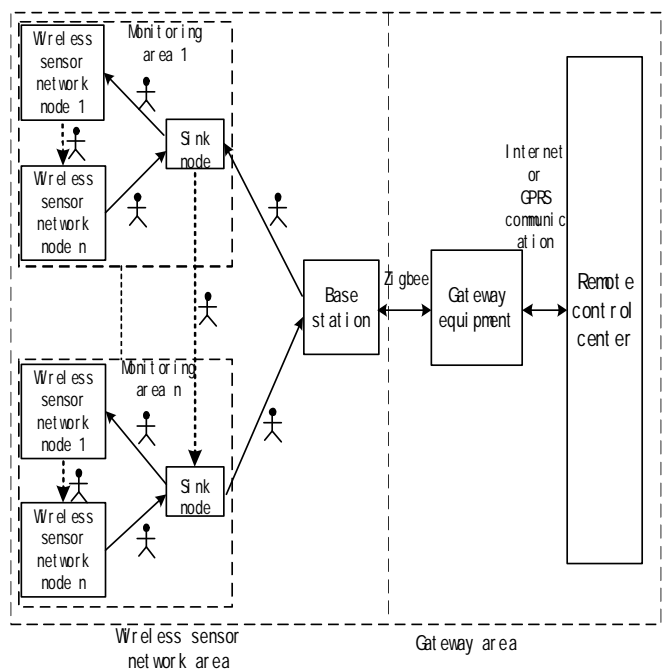

Fig. 4. Composition of the bridge structural health monitoring system

\section{B. Sensors for bridge monitoring applications}

Sensors receive the sensing signal from the monitoring area and convert it into an available input signal according to certain requirements, in order to complete the signal detection for the whole monitoring system. According to the actual bridge structural state and environmental factors, more attention has been paid to consider and analyze system load source, system characteristics and the response of bridge [11]. From multi-angle evaluation such as the practicality, economy and stability, the system is equipped with a three-axis acceleration sensor and obtains the vibration signal through the measured bridge acceleration data. In addition, sensitive components of the acceleration sensor are made of metal or semiconductor material. Large changes of ambient temperature will affect the static characteristics of acceleration sensor during work. Meanwhile, its output value will produce a greater deviation as well. Based on various factors, the system is equipped with temperature and humidity sensors and light intensity sensors for compensating the acceleration data.

\section{System design}

\section{A. System hardware design}

The hardware part of the system includes the hardware design of wireless sensor network node and gateway node. The wireless sensor network node [12] is the sensing component of health monitoring system which can obtain information of bridge structure. Generally, it consists of four parts that are sensor module, data processing module, wireless transceiver module and power module. The sensor module is responsible for collecting information of monitoring area and converting it into a digital signal, which is transmitted to the data processing module. The data processing module is responsible for completing the work of processing and storing data. The wireless transceiver module is responsible for wireless communication with other nodes, exchanging control information, sending data and receiving data. The power module provides required energy for wireless sensor nodes [13].

The composition of wireless sensor network nodes is shown in figure 5. The latest CC2530 of TI company integrates a $2.4 \mathrm{GHz}$ RF transceiver with a 8051 microprocessor, $8 \mathrm{kB}$ RAM, $32 / 64 / 128 / 256 \mathrm{~KB}$ flash, as well as other powerful support functions and peripherals. Therefore, the system chooses CC2530 chip as wireless transceiver module, which not only reduces cost and node volume, but also reduces energy consumption of network in the communication. 


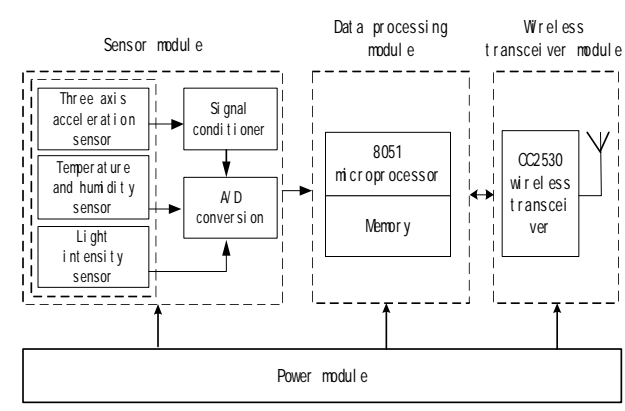

Fig. 5. Composition of wireless sensor network nodes

The gateway node is a transfer station which communicates between wireless sensor network and host computer. The gateway node is mainly composed of wireless transceiver module, data processing module, GPRS wireless communication module and power module. The wireless transceiver module transmits data sent by sensor node to data processing module. Then the data processing module sends the processed data to the remote control center through GPRS.

The composition of gateway node is shown in figure 6. ARM7 microprocessor is the mainstream embedded processor designed by a British company called ARM. Samsung Corp S3C4510B is picked in this paper with its high-level instruction set which is operated by Linux system.

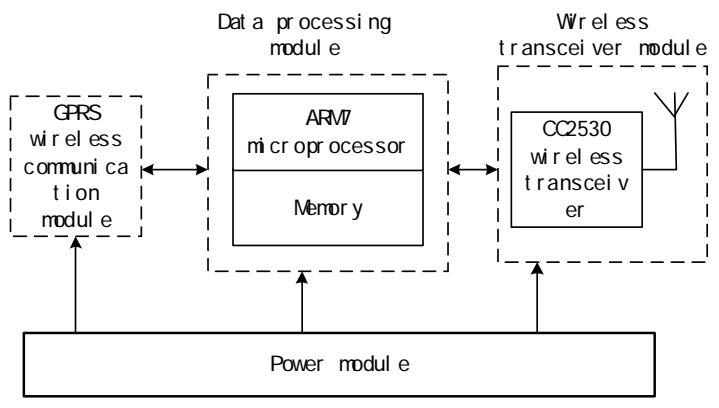

Fig. 6. Composition of gateway nodes

\section{B. System software design}

In the aspect of system software design, this paper selects Agilla as mobile agent platform which is developed by the University of Washington. This platform is a mobile agent middleware which is designed specifically for wireless sensor networks. It is applied to the rapid deployment of adaptive wireless sensor networks. Agilla allows user to join mobile agents in the network nodes. These mobile agents coordinate their work through the Tuple Space, and migrate intellectually and dynamically in the network in order to complete special tasks. Agilla operates on the TinyOS system and uses nesC programming (C language for network embedded systems).

The PC software part utilizes LabVIEW graphical programming language. The display interface of the monitoring system designed by LabVIEW can realize multi-channel data acquisition, data display, data storage and alarm function.

\section{Experiment and analysis}

\section{A. Sensor measuring point layout}

This paper takes viaduct as an example. The length of bridge is 564 meters. The width of bridge is 18.5 meters. There are 15 spans in total. According to the civil structure of bridge, the key parts of bridge are selected as the node layout and monitoring object. Respectively, nodes are arranged in the center of the sixth span, the seventh span, the ninth span and the tenth span, 1/4 center and the end of span. Each span has 5 sensor nodes and 1 Sink node. A base station is arranged in the center of the eighth span. In total, 24 nodes are arranged. The node layout diagram is shown in figure 7 . When network is constructed, the nodes can be clustered by spans. The Sink node is a cluster head, which is responsible for communication with base station. 


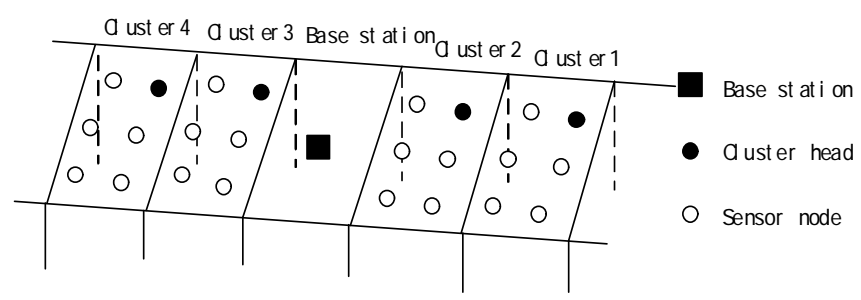

Fig. 7. Bridge node layout diagram

\section{B. Data analysis}

When the monitoring system is started, the sensor node is set for acceleration data acquisition with a frequency of $50 \mathrm{~Hz}$. The acceleration responses are recorded in the absence of vehicles and vehicles crossing the bridge respectively. These collected acceleration data are analyzed by frequency spectrum. The acceleration response of vehicles crossing the bridge and its frequency spectrum are shown in figure 8 .

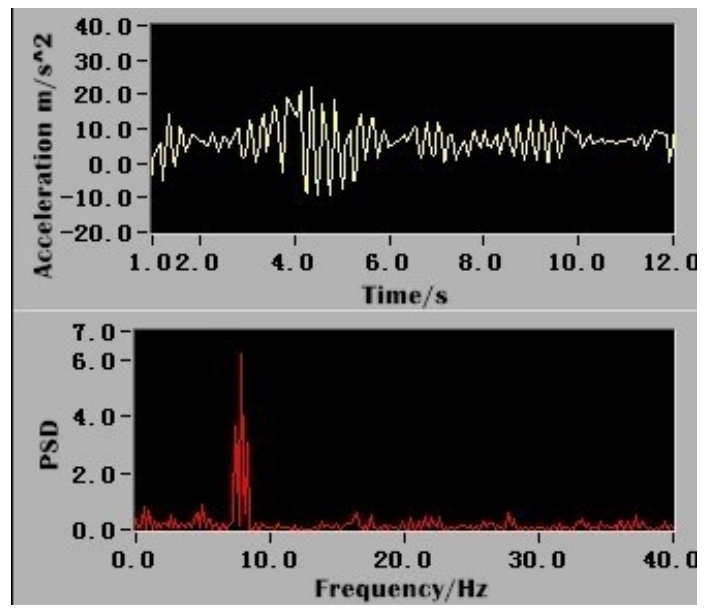

Fig. 8. Vehicles crossing the bridge acceleration response and frequency spectrum

During the detection process, it is found that bridge will vibrate when vehicles pass. Meanwhile, the vibration frequency can reflect the influence of vehicles and other external forces on the bridge. In figure 8, the maximum frequency of bridge isError! Reference source not found.. However, the theoretical value of natural frequency is Error! Reference source not found. based on the construction data of bridge structure. By analysis, difference between two values is within tolerant range. The result shows that the bridge is in good condition and it is not necessary to carry out targeted maintenance.

\section{Conclusion}

Experiments show that the effective combination of wireless sensor network and mobile agent technology has achieved good effect in bridge structure health monitoring, which is one of the best effective schemes to monitor bridge health. The system has successfully applied mobile agent technology to data acquisition. On the one hand, this measure makes the acquisition task more flexible and convenient. The resource utilization rate of system has been improved. On the other hand, the large and dense wireless sensor network can be managed effectively by the whole system. However, due to the complex diversity of bridge structure, the monitoring system needs to be further improved and perfected.

\section{Acknowledgements}

This work was financially supported by the Scientific Research Significant Projects of Universities and Colleges in Jiangsu Province (16KJA460003) 


\section{REFERENCES}

[1] Bo Yu, Hongxing Qiu, Hao Wang, et al: Journal of earthquake engineering and engineering vibration, vol.29(2009),p170.

[2] Youliang Ding, Aiqun Li: Journal of Southeast University(Natural Science Edition), vol.38(2003),p704.

[3] Lin Ji, Huaping Ding, Qinghong Shen: Journal of Nanjing University (Natural Sciences), vol.47(2011),p19.

[4] Akyildiz F, Su W, Sankarasubramaniam Y, et al: Computer Networks, vol.38(2002),p393.

[5] Ying Chen, Jian Shu, Yubin Chen, Min Yin: Transducer and Microsystem Technologies, vol.26(2007),p1.

[6] Hairong Qi, Yingyue Xu, Xiaoling Wang: Proceeding of the IEEE, vol. 91(2003),p1172.

[7] Yunyong Zhang.:Mobile agent technology and its application(Tsinghua University Press, Beijing 2003)

[8] Jietai Wang, Shaojun Yang, Haixun Yu, et al: Computer Engineering, vol.34(2008),p133.

[9] Shu Wang, Shujie Yan, Shuai Chen, et al: Chinese Journal of Sensors and Actuators, vol.19(2006)p.926.

[10]Ding H P, Feng Z X, Shen Q H, et al: Journal of Nanjing University (Natural Sciences), vol.45 (2009),p488.

[11]Changqing Miao, Aiqun Li, Xiaolin Han, et al: Journal of Southeast University(Natural Science Edition), vol.35(2005),p78.

[12]Xinhui Li, Along Yu, Miao Pan: Transducer and Microsystem Technologies, vol.32(2013),p85.

[13]Edgar H.Callaway,Jr, Yongbin Wang, Xiaoxu Qu:Wiress Sensor Networks: Architectures and Protocols (Publishing House of Electronics Industry, Beijing,2006) 